\title{
Effective Hamiltonian for FeAs-based superconductors
}

\section{Citation}

Manousakis, Efstratios, Jun Ren, Sheng Meng, and Efthimios Kaxiras. 2008. "Effective Hamiltonian for FeAs-Based Superconductors." Physical Review B 78 (20). https:// doi.org/10.1103/physrevb.78.205112.

\section{Permanent link}

http://nrs.harvard.edu/urn-3:HUL.InstRepos:41384090

\section{Terms of Use}

This article was downloaded from Harvard University's DASH repository, and is made available under the terms and conditions applicable to Open Access Policy Articles, as set forth at http:// nrs.harvard.edu/urn-3:HUL.InstRepos:dash.current.terms-of-use\#OAP

\section{Share Your Story}

The Harvard community has made this article openly available.

Please share how this access benefits you. Submit a story.

Accessibility 


\title{
Effective Hamiltonian for FeAs based superconductors
}

\author{
Efstratios Manousakis ${ }^{(1,2)}$, Jun Ren ${ }^{(3)}$, Sheng Meng ${ }^{(3)}$ and Efthimios Kaxiras ${ }^{(3)}$ \\ ${ }^{(1)}$ Department of Physics and MARTECH, Florida State University, Tallahassee, FL 32306-4350, USA \\ ${ }^{(2)}$ Department of Physics, University of Athens, Panepistimioupolis, Zografos, 15784 Athens, Greece \\ ${ }^{(3)}$ Department of Physics and School of Engineering and Applied Sciences, \\ Harvard University, Cambridge, MA 02138, USA
}

(Dated: October 22, 2018)

\begin{abstract}
The recently discovered FeAs-based superconductors show intriguing behavior and unusual dynamics of electrons and holes which occupy the Fe $d$-orbitals and As $4 s$ and $4 p$ orbitals. Starting from the atomic limit, we carry out a strong coupling expansion to derive an effective hamiltonian that describes the electron and hole behavior. The hopping and the hybridization parameters between the Fe $d$ and As $s$ and $p$-orbitals are obtained by fitting the results of our density-functional-theory calculations to a tight-binding model with nearest-neighbor interactions and a minimal orbital basis. We find that the effective hamiltonian, in the strong on-site Coulomb repulsion limit, operates on three distinct sub-spaces coupled through Hund's rule. The three sub-spaces describe different components (or subsystems): (a) one spanned by the $d_{x^{2}-y^{2}}$ Fe orbital; (b) one spanned by the degenerate atomic Fe orbitals $d_{x z}$ and $d_{y z}$; and (c) one spanned by the atomic Fe orbitals $d_{x y}$ and $d_{z^{2}}$. Each of these Hamiltonians is an extended $t-t^{\prime}-J-J^{\prime}$ model and is characterized by different coupling constants and filling factors. For the case of the undoped material the second subspace alone prefers a ground state characterized by a spin-density-wave order similar to that observed in recent experimental studies, while the other two subspaces prefer an antiferromagnetic order. We argue that the observed spin-density-wave order minimizes the ground state energy of the total hamiltonian.

PACS numbers: 74.70.-b,74.25.Ha,74.25.Jb,75.10.-b
\end{abstract}

\section{INTRODUCTION}

The recent observation of superconductivity in quaternary oxypnictides $1,2,3,4,5,6$, which are materials based on FeAs (and FeP), has rekindled intense activity $7,8,9,10,11,16,17,18,19,20,21,22,23,24,25,26,27,28$ to find a description of the strong electronic correlations present in these materials and in the cuprates which could be responsible for such phenomena.

The structure of the new materials 2 (Fig. 1) consists of FeAs layers sandwiched between $\mathrm{LaO}$ layers with rather weak interlayer coupling. The FeAs layers consist of a square lattice formed by the Fe atoms, while the As atoms, which sit just above and just below the plane (see Fig. 1), form $\mathrm{FeAs}_{4}$ octahedra squeezed along the $c$ axis such that each Fe-As bond forms a $\pm 30^{\circ}$ angle with the Fe plane.

As a function of temperature, the resistivity of the undoped parent compound, which is not an insulator, shows a drop around $150 \mathrm{~K}^{2,29}$ before turning back up below 50 $\mathrm{K}$. In addition, the magnetic susceptibility also shows an anomaly at $150 \mathrm{~K}$ and it was argued 29 that the parent material has a spin-density-wave (SDW) instability below $150 \mathrm{~K}$. Recent neutron diffraction studies 30 demonstrate that the parent compound at $150 \mathrm{~K}$ undergoes a structural distortion from tetragonal at high temperature to monoclinic at low temperature. Furthermore, these neutron diffraction studies show that below $\sim 134 \mathrm{~K}$, while in the monoclinic phase, it develops the SDW order shown in Fig. 1. Subsequent Mössbauer and muon spin rotation $(\mu S R)$ studies $^{31}$ confirmed these findings: the structural transition is found to be around $156 \mathrm{~K}$ and the magnetic transition around $138 \mathrm{~K}$. The structural distortion which brings about the monoclinic structure at low temperature is such that the rows of atoms which have their spins antiferromagnetically aligned are closer than the rows of atoms in the perpendicular direction.

The electronic structure of $\mathrm{LaO}_{1-x} \mathrm{~F}_{x} \mathrm{FeAs}$ has been studied by density functional theory ${ }^{7,11}$ and by dynamical mean field theory ${ }^{9}$. There are arguments against phonon-mediated superconductivity ${ }^{9,10}$ in $\mathrm{LaO}_{1-x} \mathrm{~F}_{x} \mathrm{FeAs}$. In addition, there are suggestions that a two-band model16,17,18,19,22 may be the right effective hamiltonian to use in order to describe the low energy physics of these materials.

Since the interlayer coupling is found to be weak ${ }^{11}$, in order to provide a simpler basis to understand the electronic structure of these materials we will focus on a single FeAs layer. As further justification of this choice, we have calculated the band structure of LaOFeAs and that of a single FeAs layer within density-functional theory and we find that the important features of the bands near the Fermi level obtained by the two calculations are essentially the same, including position relative to the Fermi level and overall dispersion features. Accordingly, our starting point is a hamiltonian which includes the five Fe $d$-orbitals and four outer As orbitals ( $4 s$ and $4 p$ ). In our interacting electron model we include direct Fe-Fe hopping, hybridization between the Fe $d$ and the As $4 s$ and $4 p$ orbitals, the local Coulomb repulsion energy for adding an electron on any of the Fe $d$ states via Hubbard-type terms in the hamiltonian, and finally, coupling through Hund's rule.

We determine the relative energy of the atomic orbitals 
as well as the hopping and the hybridization matrix elements as follows: We carry out a set of first-principles calculations of the electronic structure of a single FeAs layer. Using as basis the above mentioned Fe and As states, we fit the results of the first-principles calculations using the tight-binding approximation to determine the values of the hopping matrix elements and the on-site energy levels. We find that the energy difference between the atomic orbitals is less than $1 \mathrm{eV}$ while the Coulomb repulsion to add two electrons on the same Fe $d$ orbital is assumed to be significantly larger $11,12,13,14,15,28$. We perform a strong coupling expansion in which the hopping and hybridization terms are used as perturbation and the unperturbed parts are the terms which correspond to the Coulomb repulsion energies for a pair of electrons placed on the same or different orbitals. Even in the case where these class of materials do not fulfil the requirement for a strong coupling expansion, the qualitative results or trends suggested by such a systematic analysis may still be useful. We systematically derive a low energy effective hamiltonian to describe this multi-band system.

Through this analysis, we show that the relevant low energy degrees of freedom can be described by two 2"flavor" subsystems, one in which the two flavors correspond to electrons in the $d_{x y}$ and $d_{z^{2}}$ states of the Fe atoms and another in which the two flavors correspond to $d_{x z}$ and $d_{y z}$ Fe orbitals, and a third 1-flavor subsystem corresponding to the $d_{x^{2}-y^{2}}$ orbital. Each of those subsystems is described by a $t-t^{\prime}-J-J^{\prime}$ model where the spin degrees of freedom of all three subsystems couple through Hund's rule. Using this hamiltonian we are able to explain why the undoped material orders in the SDW pattern shown in Fig. 1] as reported by recent neutron scattering experiments 30 .

The paper is organized as follows: Section II gives a detailed discussion of our density-functional-theory band structure calculations. Section III presents the strongcoupling limit analysis which leads to the effective spinspin interaction hamiltonian derived in Section IV. Section $\mathrm{V}$ gives a discussion of the physics of the effective hamiltonian.

\section{ELECTRONIC STRUCTURE CALCULATIONS}

\section{A. First-principles electronic structure}

Our first-principles calculations are performed within the framework of density functional theory (DFT) and the local density approximation (LDA) for exchangecorrelation effects. We used the SIESTA code ${ }^{32}$ because it employs a localized basis of atomic-like orbitals for the expansion of the wavefunctions which makes the interpretation of electronic wavefunctions in the solid straightforward and transparent, without the need for additional analysis such as projection to localized Wannier-type orbitals. We use pseudopotentials of the Troullier-Martins

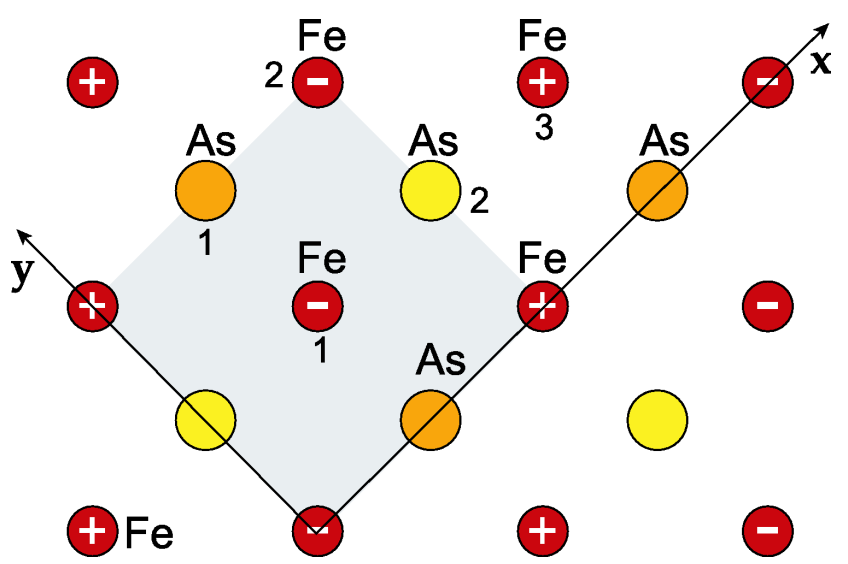

FIG. 1: The structure of the FeAs layer in the FeAs based superconductors. The Fe atoms form a square lattice while the As atoms form two square sublattices one just above (orange) and the other just below (yellow) the Fe plane. The $\mathbf{x}$ and $\mathbf{y}$ axes used to characterize the orbitals are shown and the unit cell is indicated by the shaded square. The + and - signs on the Fe atoms denote the spin orientation observed in the neutron scattering experiment ${ }^{30}$. The labels are used in the text to explain the various types of antiferromagnetic exchange interactions.

type 33 to represent the interaction between valence electrons and ionic cores, and the Ceperley-Alder form for the exchange-correlation functional ${ }^{34}$. We have generated several different $\mathrm{Fe}$ pseudopotentials in order to check for any dependence of the results on this ingredient of the calculations. We find that the effect of the different pseudopotentials on the quantities reported in the following, such as the electronic bands, is indiscernible. In addition to the SIESTA calculations, we have used the VASP code to ensure that there is reasonable agreement between the results of two very different computational schemes. The VASP code uses a plane-wave basis instead of localized orbitals 35 and employs pseudopotentials of a different type ${ }^{36}$ than those in the SIESTA calculation.

LaOFeAs, belonging to the tetragonal $\mathrm{P} 4 / \mathrm{nmm}$ space group, has a layered structure ${ }^{2}$. The FeAs layer serves as the carrier conduction channel and it has strong electronic couplings within the layer. The unit cell for the simplified model system, a single FeAs layer, contains two Fe and two As atoms with a vacuum layer with thickness $\sim 19 \AA$. The full system, bulk LaOFeAs has two atoms of each type (Fe, As, La and O) in the unit cell.

In the SIESTA calculations, we choose an auxiliary real space grid equivalent to a plane-wave cutoff of $100 \mathrm{Ry}$, and use $8 \times 8 \times 1$ Monkhorst-Pack k-point grid for the FeAs layer, $4 \times 4 \times 1$ for the $(2 \times 2)$ FeAs supercell, and a $8 \times 8 \times 4$ grid for the LaOFeAs structure. For geometry optimization, a structure is considered fully relaxed when the magnitude of forces on all atoms is smaller than 0.04 


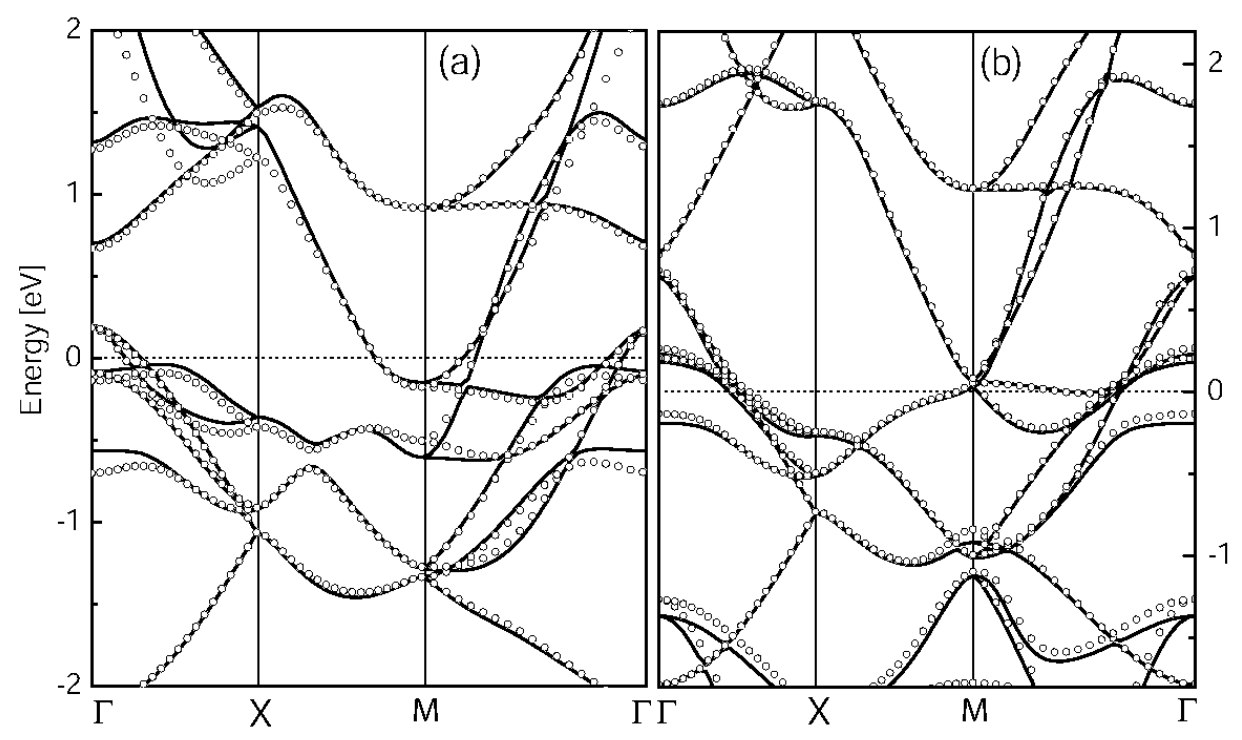

FIG. 2: The band structure obtained from the LDA calculations, for the paramagnetic phase of: (a) the LaOFeAs compound; (b) the FeAs layer. In both cases the lines are from the SIESTA calculations and the points from the VASP calculation. The Fermi level is set at zero in each case, for the neutral material.

$\mathrm{eV} / \AA$. In the VASP calculation we use the same k-point grids and the default plane-wave cutoffs.

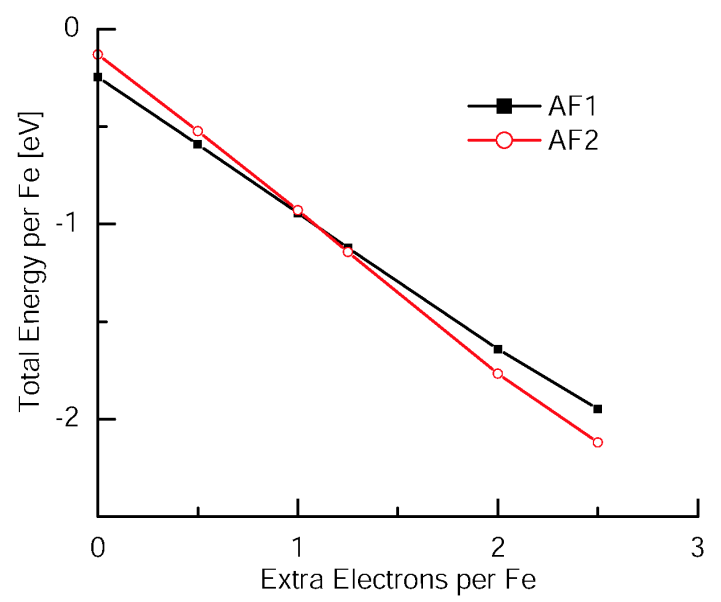

FIG. 3: The total energy of the FeAs layer as a function of the added charge obtained with the VASP code. The reference energy is the energy of the neutral layer in the paramagnetic phase.

The results obtained for bulk $\mathrm{LaOFeAs}$ and for the FeAs layer, using the SIESTA and VASP codes, for the paramagnetic phase are shown in Fig. 2. In both cases, the agreement between the two different computational schemes is remarkable. Moreover, there a very close similarity between the bands of the bulk LaOFeAs compound and the FeAs layer, especially in what concerns the features near the Fermi level. Notice that the neutral FeAs layer contains a different number of electrons per Fe atom than the bulk $\mathrm{LaOFeAs}$ compound. Accordingly, we show the Fermi levels for the bulk and the layer slightly offset, to emphasize the similarity of the band-structure features. Moreover, by examining carefully the wavefunction character of the bands near $\Gamma$ and $\mathrm{M}$, the points in the Brillouin Zone where electron and hole pockets appear in the bulk LaOFeAs compound, we establish that these states arise from orbitals associated with the Fe and As atoms. For these reasons, it is reasonable to concentrate on the band-structure of the FeAs layer alone, in order to build a comprehensive picture of the interacting electron system, presented in the following sections.

We address next the issue of the spin configuration. We considered different spin configurations in the antiferromagnetic (AFM) phase. For these calculations we use a $(2 \times 2)$ FeAs supercell. The two spin configurations are: (i) AFM1, which is simply repetition of the spin configuration in the $(1 \times 1)$ unit cell; (ii) AFM2, which has the same spin alignment in one of the two diagonal directions of the Fe lattice and alternating spins along the other direction, as shown in Fig. 1. The total energy for the former spin configuration is lower by $0.95 \mathrm{eV}$ per $(2 \times 2)$ cell, suggesting the AFM1 configuration is more stable than the AFM2 one for the neutral FeAs layer. We believe that this result is due to the fact that our calculation is for the charge neutral FeAs layer, where the Fermi level is lower than that of the LaOFeAs compound; namely, the FeAs layer as part of the LaOFeAs structure is negatively charged by an extra electron per $\mathrm{Fe}$ atom because the $\mathrm{LaO}$ layer is positively charged since the preferred oxidation state of $\mathrm{La}$ is $\mathrm{La}^{3+}$ and that of 
oxygen is $\mathrm{O}^{2-}$. To show that this is the case, we carried out a calculation using the VASP code for a charged FeAs layer. In Fig. 3 the total energies of the AFM1 and AFM2 phases are compared as a function of the added charge $\sigma$ per Fe atom. Notice that the AFM2 phase becomes energetically favorable for $\sigma \sim 1$. The exact values of $\sigma$ can not be accurately determined in the context of these calculations because the energy differences are within the range of accuracy of DFT-LDA. The fact that the AFM2 phase becomes the ground state for $\sigma=1$ will be established using the effective Hamiltonian derived in the next section, which captures the interacting-electron nature of the system in a more realistic manner, and as such, gives more reliable results for the magnetic phases.

\section{B. Tight-binding approximation model}

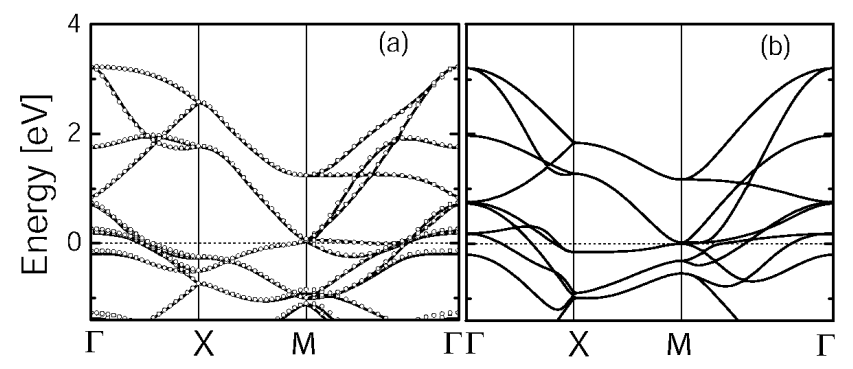

FIG. 4: The band structure of FeAs layer in the paramagnetic phase obtained from: (a) the LDA calculations with the two different approaches (SIESTA - lines, and VASP - points); (b) the tight-binding approximation with nearest neighbor interactions and minimal orbital basis.

Our goal next is to calculate a tight-binding hamiltonian which approximately gives the same band structure as that of the first-principles results for the FeAs layer, using nearest-neighbor interactions only (hopping matrix elements) and a minimal orbital basis, consisting of the
$4 s$ and $4 p$ As orbitals and the $3 d$ Fe orbitals. We expect that the hopping matrix elements, needed for the hamiltonian upon which the strong coupling expansion will be based, are not significantly affected by the value of the filling factor (the position of the Fermi level). For the reasons discussed above, namely that a realistic picture of spin configurations can only arise from the interactingelectron treatment based on the effective hamiltonian, we will focus on reproducing with the tight-binding approximation the band structure of the paramagnetic phase, as obtained from the DFT-LDA calculations.

Since introduction of electron doping is necessary in order to produce superconductivity in LaOFeAs-based materials, we focus in reproducing as accurately as possible the features near and above the Fermi level. Note that hole-doping induced superconductivity in these materials has been also reported recently ${ }^{\underline{6}}$. We also use information from the first-principles electronic wavefunctions to determine what is the optimal fit of the tight-binding approximation to the DFT-LDA results. As mentioned above, all the bands in the neighborhood of the Fermi level are associated with the As $4 s$ and $4 p$ and the $\mathrm{Fe}$ $3 d$ orbitals, and these features are well reproduced by the tight-binding approximation results. The best fit we could achieve is shown in Fig. 4, and compared to the first-principles results for an extended region near the Fermi level. The on-site and hopping matrix elements that produce this fit are presented in the next section, where these values are employed to construct the effective hamiltonian.

\section{STRONG COUPLING LIMIT}

We consider the hamiltonian describing a single FeAs layer of $\mathrm{Fe}$ and $\mathrm{As}$ (or, in a more general formulation, $\mathrm{P}$ ) atoms:

$$
\hat{H}=\hat{H}_{a}+\hat{T}+\hat{U},
$$

with the three terms defined by:

$$
\begin{aligned}
\hat{H}_{a} & =\sum_{i, \nu, \sigma} \epsilon_{d}(\nu) d_{i \sigma}^{(\nu) \dagger} d_{i \sigma}^{(\nu)}+\sum_{l, \alpha, \sigma} \epsilon_{s p}(\alpha) s p_{l \sigma}^{(\alpha) \dagger} s p_{l \sigma}^{(\alpha)}, \\
\hat{T} & =-\sum_{<i j>, \sigma, \nu \nu^{\prime}}\left(t_{\nu \nu^{\prime}} d_{j \sigma}^{\left(\nu^{\prime}\right) \dagger} d_{i \sigma}^{(\nu)}+h . c\right)-\sum_{i, \sigma, \nu, \alpha} \sum_{l(i)}\left(V_{i l}^{\nu \alpha} d_{i \sigma}^{(\nu) \dagger} s p_{l \sigma}^{(\alpha)}+h . c\right), \\
\hat{U} & =\sum_{\nu} U_{\nu}^{(d)} \sum_{i} n_{d}^{\nu}(i \uparrow) n_{d}^{\nu}(i \downarrow)+\sum_{\nu, \nu^{\prime} \neq \nu} U_{\nu \nu^{\prime}}^{(d)} \sum_{i \sigma \sigma^{\prime}} n_{d}^{\nu}(i \sigma) n_{d}^{\nu^{\prime}}\left(i \sigma^{\prime}\right) \\
& +\sum_{\alpha} U_{\alpha}^{(s p)} \sum_{i} n_{s p}^{\alpha}(i \uparrow) n_{s p}^{\alpha}(i \downarrow)+\sum_{\alpha, \alpha^{\prime} \neq \alpha} U_{\alpha \alpha^{\prime}}^{(s p)} \sum_{i \sigma \sigma^{\prime}} n_{s p}^{\alpha}(i \sigma) n_{s p}^{\alpha^{\prime}}\left(i \sigma^{\prime}\right)-\sum_{\nu, \nu^{\prime}} J_{\nu, \nu^{\prime}}^{H} \sum_{i} \vec{S}_{d}^{\nu}(i) \cdot \vec{S}_{d}^{\nu^{\prime}}(i) .
\end{aligned}
$$

We discuss the non-interacting parts, $\hat{H}_{a}$ and $\hat{T}$ of the hamiltonian first. The operator $d_{i \sigma}^{(\nu) \dagger}$ creates an electron of spin $\sigma$ on the $\nu^{\text {th }}$ Fe $d$-orbital $(\nu=1,2,3,4,5$ is the 
TABLE I: The on-site energies in $\mathrm{eV}$ for the Fe $3 d$ orbitals as determined by approximating the results of the first-principles band-structure calculation, using the tight-binding approximation discussed in Sec. III. We also include the hopping matrix elements $t_{\nu \nu}$ between two nearest neighbor Fe $d$-orbitals of the same type. The notation is explained in Sec. III]

\begin{tabular}{|l|l|l|l|l|l|}
\hline$\nu$ & 1 & 2 & 3 & 4 & 5 \\
\hline Fe 3d-orbital & $d_{x^{2}-y^{2}}$ & $d_{x z}$ & $d_{y z}$ & $d_{x y}$ & $d_{z^{2}}$ \\
\hline$\epsilon_{d}(\nu)$ & -4.6 & -4.5 & -4.5 & -4.5 & -4.2 \\
\hline$t_{\nu \nu}$ & 0.22 & 0.5 & 0.5 & 0.43 & 0.22 \\
\hline
\end{tabular}

TABLE II: Same as in Table I for the As $4 s 4 p$-orbitals.

\begin{tabular}{|l|l|l|l|}
\hline$\alpha$ & 1 & 2 & 3 \\
\hline As sp-orbital & $p_{x}$ & $p_{y}$ & $s p_{z}^{+}$ \\
\hline$\epsilon_{s p}(\alpha)$ & -4.7 & -4.7 & -5.2 \\
\hline
\end{tabular}

index that corresponds to the five $d$ Fe orbitals, $d_{x^{2}-y^{2}}$, $d_{x z}, d_{y z}, d_{x y}$, and $d_{z^{2}}$ respectively) at the site $i$ which has an energy $\epsilon_{d}(\nu)$. The operator $s p_{l \sigma}^{(\alpha) \dagger}$ creates an electron of spin $\sigma$ on the $\alpha^{t h}$ As which is one of three As orbitals. These As orbitals are formed as follows: first, because of the tetragonal symmetry, the two $4 p_{x}$ and $4 p_{y}$ orbitals remain unhybridized, while the $4 s$ and $4 p_{z}$ As states form two linear combinations $\left|s p_{z}^{ \pm}\right\rangle=a|s\rangle \pm b\left|p_{z}\right\rangle$. The LDA calculation shows that the As $s p_{z}^{-}$state together with the Fe $4 s$ form a bonding and an anti-bonding state, with the bonding state approximately $10 \mathrm{eV}$ below the Fermi level and the anti-bonding state approximately $6 \mathrm{eV}$ above the Fermi level. Therefore, these two states are not included in the tight-binding fit and the three As states included are the $4 p_{x}$ and $4 p_{y}$ orbitals and the $s p_{z}^{+}$hybrid. Thus, $\alpha=1,2,3$ corresponds to the cases of $4 p_{x}, 4 p_{y}$ and $s p_{z}^{+}$ respectively at the $l^{t h}$ As site, with site energy $\epsilon_{s p}(\alpha)$.

$V$ is the hybridization term between the Fe $3 d$ orbitals and the As orbitals. The sum over $l(i)$ means that it is over all four As sites $l$ around the $i^{t h} \mathrm{Fe}$ site. The hybridization matrix element $V_{i l}^{\nu \alpha}$ is proportional to the wave function overlap of the $\nu^{t h} \mathrm{Fe} d$-orbital and the As $\alpha^{t h} s p$-orbital. Some of these matrix elements are zero due to symmetry arguments and the most significant ones are of the order of, or less than, $1 \mathrm{eV}$, as obtained through our tight binding fit of the LDA results.

In Tables [III and in Table [II] we give the non-zero matrix elements obtained by fitting the LDA results to the tight binding model (as explained in Sec. III) which includes the five Fe $d$-states and the three As $4 s-4 p$ states for each of the two $\mathrm{Fe}$ and the two As atoms in the $\mathrm{Fe}_{2} \mathrm{As}_{2}$ unit cell, as well as the matrix elements $t_{\nu \nu^{\prime}}$ and $V^{\nu \alpha}$ between these states. In addition to the above terms, the tight-binding approximation to the LDA results gives two hopping matrix elements $t_{\nu \nu^{\prime}}$ : the first for $\nu \rightarrow d_{x z}$ and $\nu^{\prime} \rightarrow d_{y z}$, which is $t_{x z, y z}=0.54 \mathrm{eV}$, and the second for $\nu \rightarrow d_{x y}$ and $\nu^{\prime} \rightarrow d_{z^{2}}$, which is $t_{x y, z^{2}}=0.20 \mathrm{eV}$. All other hopping matrix elements are either identically equal to zero due to symmetry or negligibly small.
TABLE III: Same as in Table \ for the hybridization matrix elements between $\mathrm{Fe} 3 d$ - and As $4 s 4 p$-orbitals. The atom labels are those shown in Fig. 11 For the case of Fe atom labeled 2 in Fig. 1 the matrix elements are obtained from the same table by interchanging the labels 1 and 2 of the As orbitals and reversing the sign.

\begin{tabular}{|l|l|l|l|l|l|}
\hline$V^{\nu \alpha}$ & $1\left(d_{x^{2}-y^{2}}\right)$ & $2\left(d_{x z}\right)$ & $3\left(d_{y z}\right)$ & $4\left(d_{x y}\right)$ & $5\left(d_{z^{2}}\right)$ \\
\hline$p_{x}(1)$ & 0 & 0.1 & 0 & -0.2 & 0 \\
\hline$p_{x}(2)$ & 0.4 & -1.45 & 0 & 0 & 0.25 \\
\hline$p_{y}(1)$ & -0.4 & 0 & 1.45 & 0 & 0.25 \\
\hline$p_{y}(2)$ & 0 & 0 & -0.1 & -0.2 & 0 \\
\hline$s p_{z}^{+}(1)$ & -0.5 & 0 & 0.7 & 0 & 0.9 \\
\hline$s p_{z}^{+}(2)$ & -0.5 & 0.7 & 0 & 0 & -0.9 \\
\hline
\end{tabular}

We turn next to the interaction part, $\hat{U}$, of the hamiltonian. $n_{d}^{\nu}(i \sigma)=d_{i \sigma}^{(\nu \dagger \dagger} d_{i \sigma}^{(\nu)}$ is the number operator and $U_{\nu}^{(d)}$ or $U_{\alpha}^{(s p)}$ give the Coulomb repulsion for a pair of electrons placed on the same $d$-orbital or the same $s$ or $p$ As-orbital. The $U_{\nu \nu^{\prime}}^{(d)}\left(\right.$ or $U_{\alpha \alpha^{\prime}}^{(s p)}$ )is responsible for the Coulomb repulsion between different Fe- $d$ (or As $s p$ ) orbitals within the same atom. We will assume that the Coulomb-repulsion terms between the same or different $\mathrm{Fe}-d$ orbitals are significantly greater than their counterparts for the As $s p$ states, consistent with the general expectations for these values in the literature: $U^{(d)}$ is believed to be large of the order of $4-5 \mathrm{eV}^{11,12,13,14,15,28}$ while the parameter $U^{(s p)}$ is expected to be much smaller than that. In addition, we will assume that the same site Fe- $d$ Coulomb repulsion is larger than the inter-orbital Coulomb repulsion $U_{\nu \nu^{\prime}}^{(d)}$. The term proportional to $J^{H}$ represents Hund's rule for the Fe d orbitals, with $J^{H}>0$, of order of less than $1 \mathrm{eV}$. We have neglected the Hund's rule coupling for As orbitals.

Notice that the energy levels $\epsilon_{d}(\nu)$ and $\epsilon_{s p}(\nu)$ lie in the region $-4.7 \pm 0.5 \mathrm{eV}$, namely the energy difference between any pair of such states is less than $1 \mathrm{eV}$ which is believed to be smaller than the characteristic Coulomb repulsion energy $U^{(d)}$. In this paper we begin our analysis from the atomic or strong coupling limit, which implies that we have assumed that the energy scale $U^{*}$ defined below in Eq. 17 is significantly larger than the hopping and hybridization matrix elements. If this condition is not fulfilled for this class of materials, it may still be instructive to discuss the qualitative features which a strong coupling expansion yields. In this limit the unperturbed part of the hamiltonian $\hat{H}_{0}$ is

$$
\hat{H}_{0}=\hat{H}_{a}+\hat{U}
$$

and the hopping part $\hat{T}$, which includes the hybridization, plays the role of perturbation. As discussed previously, the FeAs layer in the undoped $\mathrm{LaOFeAs}$ parent compound has an additional electron relative to the neutral FeAs layer. This is due to the fact that LaO layer is expected to be in combined $1+$ oxidation state. Therefore, the eight states considered above (five Fe $d$ states 
plus three As $s p$ states) are occupied by 12 electrons. The atomic configuration is shown in Fig. 5. Since we have five more electrons than levels, five energy levels must be doubly occupied. Double occupancy of the Fe $d$ orbitals is much more costly compared to the As $s p$ orbitals; therefore, all three of the As orbitals must be doubly occupied and the only doubly occupied Fe $d$ orbital is $d_{x^{2}-y^{2}}$, which has the lowest energy. The other four Fe $d$ orbitals are singly occupied and the spin of these electrons are parallel because of Hund's rule, represented by the coupling $J_{H}$.

$\mathrm{Fe}$
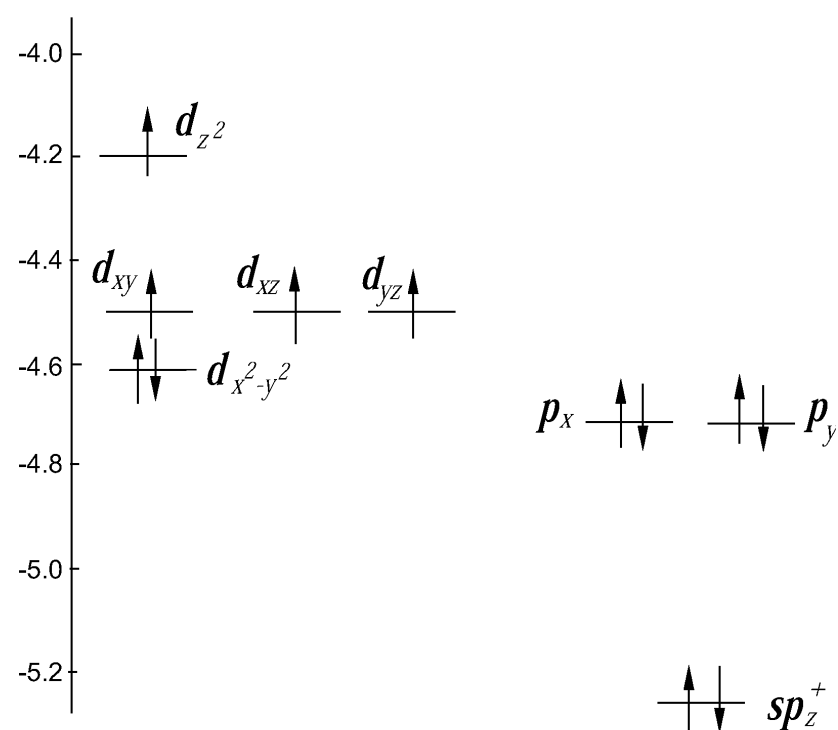

FIG. 5: The occupation of the Fe and As atomic levels in the FeAs plane of the undoped parent compound. The ordering of the levels is shown schematically, as obtained from the tightbinding approximation parameters.

Next we consider the effective hamiltonian which, in strong coupling perturbation theory, is given by

$$
\begin{aligned}
\hat{H}_{e f f} & =E_{0} \hat{P}_{0}+\hat{P}_{0} \hat{T} \hat{\Omega}, \\
\hat{\Omega} & =\hat{P}_{0}+\hat{R}(\hat{T} \hat{\Omega}-\hat{\Omega} \hat{T} \hat{\Omega}), \\
\hat{R} & =\frac{\hat{Q}}{\hat{H}_{0}-E_{0}},
\end{aligned}
$$

where $E_{0}$ is the ground state energy given by the energy

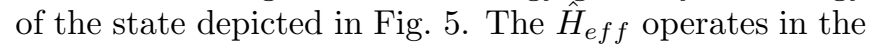
subspace $S_{0}$ formed by the degenerate ground states of $\hat{H}_{0}$, that is, the subspace of states produced by the direct product of atomic states like those in Fig. 5 in which the spins of the four electrons occupying the four $d$-orbitals, from one Fe atom to the next, point either all up or all down. The operator $\hat{P}_{0}$ is a projection operator which projects into the subspace $S_{0}$ and $\hat{Q}=\hat{1}-\hat{P}_{0}$, that is, the operator which projects outside the subspace $S_{0}$. The above equation can be formally solved iteratively to yield the Rayleigh-Schrödinger expansion as a power series in $\hat{T}$. The leading term is $\hat{T}$ which, when restricted in this subspace $S_{0}$, becomes just the direct Fe-Fe hopping terms

$$
\hat{P}_{0} \hat{T} \hat{P}_{0}=-\sum_{<i j>, \sigma, \nu \nu^{\prime}}\left(t_{\nu \nu^{\prime}} d_{j \sigma}^{\left(\nu^{\prime}\right) \dagger} d_{i \sigma}^{(\nu)}+h . c\right),
$$

In the right-hand-side of the above equation we have omitted the projection operators by assuming that we will restrict ourselves to the subspace $S_{0}$.

In the following, in order to simplify the calculation, we will take $U_{\nu}^{(d)}=U$ to be independent of $\nu$ and we will assume that $U$ is much larger than the atomic energy level difference (which was found to be less than $1 \mathrm{eV}$ within our TB approximation) and significantly larger than the hopping and hybridization parameters. In addition, we will take $U_{\nu \neq \nu^{\prime}}^{(d)}=\bar{U}, U_{\nu}^{(s p)}=U_{s p}$ and $U_{\alpha \neq \alpha^{\prime}}^{(s p)}=\bar{U}_{s p}$, i.e., to be independent of $\nu, \nu^{\prime}$ (or $\alpha, \alpha^{\prime}$ ).

\section{EFFECTIVE SPIN-SPIN INTERACTION HAMILTONIAN}

\section{A. Interaction between same-type Fe orbitals}

First, there are the familiar second order processes arising from the direct Fe-Fe hopping through the matrix elements $t_{\nu \nu}$ which give rise to an antiferromagnetic exchange interaction of the form

$$
\begin{aligned}
\mathcal{H}_{\nu, \nu} & =J_{\nu, \nu}^{(2)} \sum_{<i j>} \vec{S}_{i}^{\nu} \cdot \vec{S}_{j}^{\nu}, \\
J_{\nu, \nu}^{(2)} & =\frac{4 t_{\nu, \nu}^{2}}{U}, .
\end{aligned}
$$

In addition, we have antiferromagnetic coupling of the spins of two different type Fe $d$-orbitals due to the hopping terms $t_{x z, y z}$ and $t_{x y, z^{2}}$ which give rise to

$$
\begin{aligned}
\mathcal{H}_{\nu, \nu^{\prime}} & =J_{\nu, \nu^{\prime}}^{(2)} \sum_{<i j>} \vec{S}_{i}^{\nu} \cdot \vec{S}_{j}^{\nu^{\prime}}, \\
J_{\nu, \nu^{\prime}}^{(2)} & =\frac{4 t_{\nu, \nu^{\prime}}^{2}}{U}
\end{aligned}
$$

where $\nu, \nu^{\prime}$ can be either $x z, y z$ or $x y, z^{2}$. These processes take place only between nearest neighbors such as the Fe atoms 1 and 2 in Fig. 1 These second order contributions are obtained from the square of the matrix elements listed in Table IV by multiplying them with $4 / U$. There are no second-order nnn contributions to the spin-spin interaction.

The next terms to leading order, beyond the first and second order terms discussed above, are fourth order processes involving an $\mathrm{Fe} d$-orbital and the As $s p$-orbitals 
TABLE IV: The second-order nn contribution to the spin-spin couplings is obtained from the matrix elements listed below (in units of $\mathrm{eV}^{2}$ ) by multiplying them with $4 / U$.

\begin{tabular}{|l|l|l|l|l|l|}
\hline$t_{\nu \nu^{\prime}}^{2}$ & $d_{x^{2}-y^{2}}$ & $d_{x z}$ & $d_{y z}$ & $d_{x y}$ & $d_{z^{2}}$ \\
\hline$d_{x^{2}-y^{2}}$ & 0.048 & 0 & 0 & 0 & 0 \\
\hline$d_{x z}$ & 0 & 0.25 & 0.29 & 0 & 0 \\
\hline$d_{y z}$ & 0 & 0.29 & 0.25 & 0 & 0 \\
\hline$d_{x y}$ & 0 & 0 & 0 & 0.185 & 0.04 \\
\hline$d_{z^{2}}$ & 0 & 0 & 0 & 0.04 & 0.048 \\
\hline
\end{tabular}

with which it hybridizes. The contribution of all $s p$ orbitals of the two possible intervening As atoms to the exchange interaction between the same $d$ orbital of two nn Fe atoms is given by ${ }^{37}$

$$
\begin{aligned}
\mathcal{H}_{\nu, \nu} & =J_{\nu, \nu}^{(4)} \sum_{<i j>} \vec{S}_{i}^{(\nu)} \cdot \vec{S}_{j}^{(\nu)}, \\
J_{\nu, \nu}^{(4)} & =\sum_{\alpha=1}^{6} J_{\nu \nu}^{\alpha}, \\
J_{\nu \nu}^{\alpha} & =2 b^{2}\left[\frac{1}{U^{*}}+\frac{1}{U^{*}+\epsilon}\right], \quad b=\frac{V_{1}^{\alpha \nu} V_{2}^{\alpha \nu}}{U^{*}+\epsilon},
\end{aligned}
$$

where

$$
U^{*}=U+5 \bar{U}-\left(4 \bar{U}_{s p}+U_{s p}\right)
$$

and $U^{*}$ is assumed significantly larger that all other energy scales in the problem. Here

$$
\epsilon=\epsilon_{s p}(\alpha)-\epsilon_{d}(\nu)
$$

is the energy difference between the As $s p$ state and that of the Fe $d$ orbital. The subscripts 1 and 2 in the matrix elements refer to the fact that the two nn atoms 1 and 2 are at $90^{\circ}$ angle relative to the position of the intervening As atom and, therefore, $V_{1}$ is from Table III, while $V_{2}$ from Table III by interchanging the index 1 and 2 and the sign of the matrix elements.

Since $\epsilon<1 \mathrm{eV}$ for any combination of Fe- $d$ and the three As $s p$ orbitals and $U^{*} \sim 5 \mathrm{eV}$, if $\epsilon$ is neglected in the above expression the error in our estimate for the exchange couplings $J$ will be rather small. We are going to use the full expression given by Eq. 16 when we compute the coupling constants $J$ between the same-type Fe orbitals. We will also use this approximation of neglecting $\epsilon$ because it simplifies the results and this gives additional insight. With this approximation, we find that

$$
\begin{aligned}
& J_{\nu, \nu}^{(4)} \simeq \frac{4 A_{\nu \nu}}{U^{* 3}} \\
& A_{\nu \nu}=\sum_{\alpha=1}^{6}\left(V_{1}^{\alpha \nu} V_{2}^{\alpha \nu}\right)^{2} .
\end{aligned}
$$

Since the value of $U^{*}$ is not known, we can use the expression given by Eq. 20 as a measure of the relative spin-exchange interaction coupling. Namely, to obtain the actual values of $J_{\nu \nu}^{(4)}$, and the $J_{\nu \nu^{\prime}}^{(4)}$ with $\nu \neq \nu^{\prime}$ to be discussed next, we just need to multiply the values given in Table $\mathrm{V}$ by $4 /\left(U^{*}\right)^{3}$. Therefore, for two nn Fe atoms, such as Fe atoms 1 and 2 in Fig. 1, the spin-spin interaction coupling constant $J_{\nu \nu}$ is obtained by adding the second and fourth order contributions $J_{\nu \nu}^{(2)}$ and $J_{\nu \nu}^{(4)}$ respectively.

On the other hand, for two next nn Fe atoms, such as 1 and 3 in Fig. 1, there are no second order processes since there are no direct hopping between such atoms. The fourth order superexchange contributions is obtained as follows:

$$
\begin{aligned}
\mathcal{H}_{\nu, \nu}^{\prime} & =J_{\nu, \nu}^{\prime} \sum_{<<i j>>} \vec{S}_{i}^{(\nu)} \cdot \vec{S}_{j}^{(\nu)}, \\
J_{\nu, \nu}^{\prime} & =\frac{1}{2} \sum_{\alpha=1}^{6} J_{\nu \nu}^{\prime \alpha}, \\
J_{\nu \nu}^{\prime \alpha} & =b^{\prime 2}\left[\frac{1}{U^{*}}+\frac{1}{U^{*}+\epsilon}\right], \quad b^{\prime}=2 \frac{\left(V_{1}^{\alpha \nu}\right)^{2}}{U^{*}+\epsilon} .
\end{aligned}
$$

Notice, that in this case the same matrix elements $V_{1}$ are involved because both atoms participating in the superexchange form the same angle with the intervening As atoms. In addition, there is a factor of 2 difference between the above expression and Eq. 16 because there is only one possible intervening As atom for two fixed $\mathrm{Fe}$ atoms. The simplified expression, when $\epsilon$ is neglected is the following:

$$
\begin{aligned}
& J_{\nu, \nu}^{\prime(4)} \simeq \frac{4 A_{\nu \nu}^{\prime}}{U^{*} 3} \\
& A_{\nu \nu}^{\prime}=\frac{1}{2} \sum_{\alpha=1}^{6}\left(V_{1}^{\alpha \nu}\right)^{4}
\end{aligned}
$$

The values of the constant $A_{\nu \nu}^{\prime}$ are given as the diagonal matrix elements of Table VI

\section{B. Interaction between different-type Fe orbitals}

There is an effective spin-spin interaction between certain Fe orbitals of different type, $J_{\nu \nu^{\prime}}$ with $\nu \neq \nu^{\prime}$. This type of nn and nnn spin-spin interaction for most of the orbitals is significantly smaller than the $J_{\nu \nu}$ coupling constants between the same orbitals. To obtain an estimate of these we use the simplified expressions where $\epsilon$ is neglected. Namely,

$$
\begin{aligned}
& J_{\nu, \nu^{\prime}}^{(4)} \simeq \frac{4 A_{\nu \nu^{\prime}}}{U^{* 3}} \\
& A_{\nu \nu^{\prime}}=\sum_{\alpha=1}^{6}\left(V_{1}^{\alpha \nu} V_{2}^{\alpha \nu^{\prime}}\right)^{2} \\
& J_{\nu, \nu^{\prime}}^{\prime(4)} \simeq \frac{4 A_{\nu \nu^{\prime}}^{\prime}}{U^{* 3}}
\end{aligned}
$$


TABLE V: Fourth-order sum of matrix elements contributing to the nn spin-spin couplings.

\begin{tabular}{|l|l|l|l|l|l|}
\hline$A_{\nu \nu^{\prime}}$ & $d_{x^{2}-y^{2}}$ & $d_{x z}$ & $d_{y z}$ & $d_{x y}$ & $d_{z^{2}}$ \\
\hline$d_{x^{2}-y^{2}}$ & 0.13 & 0.12 & 0.12 & 0.01 & 0.40 \\
\hline$d_{x z}$ & 0.12 & 0.04 & 0.24 & 0.08 & 0.40 \\
\hline$d_{y z}$ & 0.12 & 0.24 & 0.04 & 0.08 & 0.40 \\
\hline$d_{x y}$ & 0.01 & 0.08 & 0.08 & 0 & 0.01 \\
\hline$d_{z^{2}}$ & 0.40 & 0.40 & 0.40 & 0.01 & 1.31 \\
\hline
\end{tabular}

TABLE VI: Fourth-order sum of matrix elements contributing to the nnn spin-spin couplings.

\begin{tabular}{|l|l|l|l|l|l|}
\hline$A_{\nu \nu^{\prime}}^{\prime}$ & $d_{x^{2}-y^{2}}$ & $d_{x z}$ & $d_{y z}$ & $d_{x y}$ & $d_{z^{2}}$ \\
\hline$d_{x^{2}-y^{2}}$ & 0.18 & 0.46 & 0.46 & 0 & 0.42 \\
\hline$d_{x z}$ & 0.46 & 4.66 & 0 & 0 & 0.53 \\
\hline$d_{y z}$ & 0.46 & 0 & 4.66 & 0 & 0.53 \\
\hline$d_{x y}$ & 0 & 0 & 0 & 0 & 0 \\
\hline$d_{z^{2}}$ & 0.42 & 0.53 & 0.53 & 0 & 1.32 \\
\hline
\end{tabular}

$$
A_{\nu \nu^{\prime}}^{\prime}=\frac{1}{2} \sum_{\alpha=1}^{6}\left(V_{1}^{\alpha \nu} V_{1}^{\alpha \nu^{\prime}}\right)^{2} .
$$

The values of the constants $A_{\nu \nu^{\prime}}$ and $A_{\nu \nu^{\prime}}^{\prime}$ are given as the off-diagonal matrix elements of Tables $\mathrm{V}$ and VI.

\section{Hopping between same-type Fe orbitals}

There are contributions to the effective hopping matrix elements due to second order processes. Namely, processes in which an electron from a doubly occupied As orbital momentarily hops to the nn Fe $d$ orbital and then an electron from the doubly occupied doped $d$-orbital hops to the singly occupied As orbital left behind. These processes give rise to the following expression

$$
\delta t_{\nu, \nu}=\sum_{\alpha=1}^{6} \frac{V_{1}^{\alpha \nu} V_{2}^{\alpha \nu}}{U^{*}+\epsilon},
$$

where $\epsilon$ is given by Eq. 18. Again, since the value of $U^{*}$ is not known, for large enough values of $U^{*}(\epsilon<1 \mathrm{eV})$ we can neglect $\epsilon$ in the above expression to obtain the following expression

$$
\begin{aligned}
\delta t_{\nu, \nu} & =\frac{B_{\nu \nu}}{U^{*}} \\
B_{\nu \nu} & =\sum_{\alpha=1}^{6} V_{1}^{\alpha \nu} V_{2}^{\alpha \nu}
\end{aligned}
$$

and the values of $B_{\nu \nu}$ are given as the diagonal elements in in Table VII] The actual estimates for $\delta t_{\nu \nu}$ can be obtained by dividing the values in the table by $U^{*}$. The total effective nn hopping is given as

$$
\tilde{t}_{\nu \nu}=t_{\nu \nu}+\delta t_{\nu \nu}
$$

TABLE VII: Second-order terms contributing to the effective nn hopping.

\begin{tabular}{|l|l|l|l|l|l|}
\hline$B_{\nu \nu^{\prime}}$ & $d_{x^{2}-y^{2}}$ & $d_{x z}$ & $d_{y z}$ & $d_{x y}$ & $d_{z^{2}}$ \\
\hline$d_{x^{2}-y^{2}}$ & -0.50 & 0.31 & 0.31 & 0 & 0 \\
\hline$d_{x z}$ & 0.31 & 0.29 & -0.49 & -0.29 & -0.65 \\
\hline$d_{y z}$ & 0.31 & -0.49 & 0.29 & 0.29 & 0.65 \\
\hline$d_{x y}$ & 0 & -0.29 & 0.29 & 0 & 0.1 \\
\hline$d_{z^{2}}$ & 0 & -0.65 & 0.65 & 0.01 & 1.62 \\
\hline
\end{tabular}

TABLE VIII: Second-order terms contributing to the effective nnn hopping.

\begin{tabular}{|l|l|l|l|l|l|}
\hline$B_{\nu \nu^{\prime}}^{\prime}$ & $d_{x^{2}-y^{2}}$ & $d_{x z}$ & $d_{y z}$ & $d_{x y}$ & $d_{z^{2}}$ \\
\hline$d_{x^{2}-y^{2}}$ & 0.82 & -0.93 & -0.93 & 0 & 0 \\
\hline$d_{x z}$ & -0.93 & 2.60 & 0 & -0.02 & -0.99 \\
\hline$d_{y z}$ & -0.93 & 0 & 2.60 & 0.02 & 0.99 \\
\hline$d_{x y}$ & 0 & -0.02 & 0.02 & 0.08 & 0 \\
\hline$d_{z^{2}}$ & 0 & -0.99 & 0.99 & 0 & 1.74 \\
\hline
\end{tabular}

In the case of next nn such as the Fe atoms 1 and 3 in Fig 1] we obtain

$$
\begin{aligned}
\tilde{t}_{\nu \nu}^{\prime} & =\frac{B_{\nu \nu}^{\prime}}{U^{*}}, \\
B_{\nu \nu}^{\prime} & =\frac{1}{2} \sum_{\alpha=1}^{6}\left(V_{1}^{\alpha \nu}\right)^{2} .
\end{aligned}
$$

The values of $B_{\nu \nu}^{\prime}$ are given as the diagonal elements of Table VIII.

\section{Hopping between different-type Fe orbitals}

There is a second order process by means of which a doubly occupied site can effectively hop to a nn Fe $d$ orbital of different type by involving an intervening As $s p$ orbital. These contributions are smaller than those connecting two of the same-type Fe $d$ orbitals and they can be approximated by

$$
\begin{aligned}
\delta t_{\nu, \nu^{\prime}} & =\frac{B_{\nu \nu^{\prime}}}{U^{*}} \\
B_{\nu \nu^{\prime}} & =\sum_{\alpha=1}^{6} V_{1}^{\alpha \nu} V_{2}^{\alpha \nu^{\prime}} .
\end{aligned}
$$

The values of $B_{\nu \nu^{\prime}}$ are given by the off-diagonal elements of Table VII.

There is also a second order process which gives rise to hopping between nnn Fe $d$ orbitals of different type. For this case we obtain

$$
\begin{aligned}
\delta t_{\nu, \nu^{\prime}}^{\prime} & =\frac{B_{\nu \nu^{\prime}}^{\prime}}{U^{*}} \\
B_{\nu \nu^{\prime}}^{\prime} & =\sum_{\alpha=1}^{6} V_{1}^{\alpha \nu} V_{1}^{\alpha \nu^{\prime}} .
\end{aligned}
$$


TABLE IX: The estimated matrix elements for the $J_{\nu \nu^{\prime}}$ and $J_{\nu \nu^{\prime}}^{\prime}$ for $U=5 \mathrm{eV}$.

\begin{tabular}{|l|l|l|l|l|l|}
\hline$J$ & $d_{x^{2}-y^{2}}$ & $d_{x z}$ & $d_{y z}$ & $d_{x y}$ & $d_{z^{2}}$ \\
$J^{\prime}$ & & & & & \\
\hline$d_{x^{2}-y^{2}}$ & 0.04 & 0.0 & 0.0 & 0.0 & 0.01 \\
& 0 & 0 & 0.01 & 0.01 & 0.01 \\
\hline$d_{x z}$ & 0.0 & 0.20 & 0.24 & 0.0 & 0.01 \\
& 0.01 & 0.07 & 0 & 0.0 & 0.01 \\
\hline$d_{y z}$ & 0.0 & 0.24 & 0.20 & 0.0 & 0.01 \\
& 0.01 & 0 & 0.07 & 0.0 & 0.01 \\
\hline$d_{x y}$ & 0.0 & 0.0 & 0.0 & 0.15 & 0.03 \\
& 0 & 0 & 0 & 0 & 0 \\
\hline$d_{z^{2}}$ & 0.01 & 0.01 & 0.01 & 0.03 & 0.08 \\
& 0.01 & 0.01 & 0.01 & 0.0 & 0.02 \\
\hline
\end{tabular}

The values of $B_{\nu \nu^{\prime}}^{\prime}$ are given by the off-diagonal elements of Table VIII

The total effective nn and nnn hopping for $\nu \neq \nu^{\prime}$ are given as

$$
\begin{aligned}
& \tilde{t}_{\nu \nu^{\prime}}=t_{\nu \nu^{\prime}}+\delta t_{\nu \nu^{\prime}}, \\
& \tilde{t}_{\nu \nu^{\prime}}^{\prime}=\delta t_{\nu \nu^{\prime}}^{\prime} .
\end{aligned}
$$

\section{E. Effective Hamiltonian}

In summary the effective hamiltonian is given by

$$
\mathcal{H}_{\text {eff }}=\sum_{\nu, \nu^{\prime}} \mathcal{H}_{\nu \nu^{\prime}}-J^{H} \sum_{i, \mu, \mu^{\prime}} \vec{S}_{i}^{\mu} \cdot \vec{S}_{i}^{\mu^{\prime}}
$$

where each of the $\mathcal{H}_{\nu, \nu^{\prime}}$ terms above may be written as

$$
\begin{aligned}
\mathcal{H}_{\nu, \nu^{\prime}} & =-\sum_{<i j>, \sigma} \tilde{t}_{\nu, \nu^{\prime}} c_{j \nu^{\prime} \sigma}^{\dagger} c_{i \nu \sigma}-\sum_{<<i j>>, \sigma} \tilde{t}_{\nu, \nu^{\prime}}^{\prime} c_{j \nu^{\prime} \sigma}^{\dagger} c_{i \nu \sigma} \\
& +\sum_{<i j>} J_{\nu, \nu^{\prime}} \vec{S}_{i}^{\nu} \cdot \vec{S}_{j}^{\nu^{\prime}}+\sum_{<<i j>>} J_{\nu, \nu^{\prime}}^{\prime} \vec{S}_{i}^{\nu} \cdot \vec{S}_{j}^{\nu^{\prime}} \cdot
\end{aligned}
$$

Next, we will provide estimates of the coupling constants $\tilde{t}_{\nu, \nu^{\prime}}, \tilde{t}_{\nu, \nu^{\prime}}^{\prime}, J_{\nu, \nu^{\prime}}$ and $J_{\nu, \nu^{\prime}}^{\prime}$ involved in the above model based on the values of the parameters obtained from fitting the LDA results to the tight binding model. The matrix elements for $n n$ hopping $\tilde{t}_{\nu \nu^{\prime}}$ and spin-spin interaction $J_{\nu \nu^{\prime}}$ as well as their counterparts for next nn interactions, that is, between sites diagonally across in the square lattice formed by the Fe atoms, $\tilde{t}_{\nu \nu^{\prime}}^{\prime}$ and $J_{\nu \nu^{\prime}}^{\prime}$, are given in Tables [X]X] for $U^{*}=U=5 \mathrm{eV}$ and in Tables XIXII using $U_{\nu}^{*}=U=3 \mathrm{eV}$. A more simplified model than the one given above is discussed in the following section.

\section{DISCUSSION}

First, by examining the Tables IXIXIXIXII, we notice that to a reasonable degree of approximation the following three subspaces couple with each other rather weakly:
TABLE X: The estimated matrix elements for the $t_{\nu \nu^{\prime}}$ and $t_{\nu \nu^{\prime}}^{\prime}$ for $U=5 \mathrm{eV}$.

\begin{tabular}{|l|l|l|l|l|l|}
\hline $\begin{array}{l}\tilde{t} \\
\tilde{t}^{\prime}\end{array}$ & $d_{x^{2}-y^{2}}$ & $d_{x z}$ & $d_{y z}$ & $d_{x y}$ & $d_{z^{2}}$ \\
\hline$d_{x^{2}-y^{2}}$ & 0.12 & 0.06 & 0.06 & 0 & 0 \\
& 0.08 & -0.09 & -0.09 & 0 & 0 \\
\hline$d_{x z}$ & 0.06 & 0.56 & 0.44 & -0.06 & -0.13 \\
& -0.09 & 0.26 & 0 & 0 & -0.1 \\
\hline$d_{y z}$ & 0.06 & 0.44 & 0.56 & 0.06 & 0.13 \\
& -0.09 & 0 & 0.26 & 0 & 0.1 \\
\hline$d_{x y}$ & 0 & -0.06 & 0.06 & 0.43 & 0.22 \\
& 0 & 0 & 0 & 0.01 & 0 \\
\hline$d_{z^{2}}$ & 0 & -0.13 & 0.13 & 0.22 & 0.54 \\
& 0 & -0.1 & -0.1 & 0 & 0.17 \\
\hline
\end{tabular}

TABLE XI: The estimated matrix elements for the $J_{\nu \nu^{\prime}}$ and $J_{\nu \nu^{\prime}}^{\prime}$ for $U=3 \mathrm{eV}$.

\begin{tabular}{|l|l|l|l|l|l|}
\hline$J$ & $d_{x^{2}-y^{2}}$ & $d_{x z}$ & $d_{y z}$ & $d_{x y}$ & $d_{z^{2}}$ \\
$J^{\prime}$ & & & & & \\
\hline$d_{x^{2}-y^{2}}$ & 0.08 & 0.02 & 0.02 & 0 & 0.06 \\
& 0.01 & 0.03 & 0.03 & 0 & 0.03 \\
\hline$d_{x z}$ & 0.02 & 0.34 & 0.42 & 0.01 & 0.06 \\
& 0.03 & 0.35 & 0 & 0 & 0.04 \\
\hline$d_{y z}$ & 0.02 & 0.42 & 0.34 & 0.01 & 0.06 \\
& 0.03 & 0 & 0.35 & 0 & 0.04 \\
\hline$d_{x y}$ & 0 & 0.01 & 0.01 & 0.25 & 0.05 \\
& 0 & 0 & 0 & 0 & 0 \\
\hline$d_{z^{2}}$ & 0.06 & 0.06 & 0.06 & 0.05 & 0.26 \\
& 0.03 & 0.04 & 0.04 & 0 & 0.10 \\
\hline
\end{tabular}

(a) one spanned by the $d_{x^{2}-y^{2}}$ Fe orbital; (b) one spanned by the degenerate atomic Fe orbitals $d_{x z}$ and $d_{y z} ;$ (c) one spanned by the atomic Fe orbitals $d_{x y}$ and $d_{z^{2}}$. Notice that the most significant off-diagonal matrix elements are those which couple the $d_{x z}$ and the $d_{y z}$ orbital and those which couple the $d_{x y}$ to the $d_{z^{2}}$ orbital. There are other smaller off-diagonal matrix elements which couple these subspaces weakly. These three subspaces, however, are much more strongly coupled through $J^{H}$.

We consider the undoped (LaOFeAs) case first. Because six electrons should occupy the five Fe $d$ orbitals (see Fig. 5), the lowest energy subspace spanned by $d_{x^{2}-y^{2}}$ is occupied by two electrons, and also each of the other two subspaces is also occupied by two electrons. As mentioned above, these three subspaces are coupled mainly because $J^{H} \neq 0$. Furthermore, the bands formed in any given subspace are intersected by the bands formed in the other two subspaces because their atomic energy difference is small compared to their bandwidth.

There is a great degree of magnetic frustration, as noted in Refs. 20,21,25], especially in the subspace spanned by $d_{x z} / d_{y z}$. In this subspace the next nn 
TABLE XII: The estimated matrix elements for the $t_{\nu \nu^{\prime}}$ and $t_{\nu \nu^{\prime}}^{\prime}$ for $U=3 \mathrm{eV}$.

\begin{tabular}{|l|l|l|l|l|l|}
\hline $\begin{array}{l}\tilde{t} \\
\tilde{t}^{\prime}\end{array}$ & $d_{x^{2}-y^{2}}$ & $d_{x z}$ & $d_{y z}$ & $d_{x y}$ & $d_{z^{2}}$ \\
\hline$d_{x^{2}-y^{2}}$ & 0.05 & 0.10 & 0.10 & 0 & 0 \\
& 0.14 & -0.16 & -0.16 & 0 & 0 \\
\hline$d_{x z}$ & 0.10 & 0.60 & 0.38 & -0.10 & -0.22 \\
& -0.16 & 0.43 & 0 & 0 & -0.17 \\
\hline$d_{y z}$ & 0.10 & 0.38 & 0.60 & 0.10 & 0.22 \\
& -0.16 & 0 & 0.43 & 0 & 0.17 \\
\hline$d_{x y}$ & 0 & -0.10 & 0.10 & 0.43 & 0.23 \\
& 0 & 0 & 0 & 0.01 & 0 \\
\hline$d_{z^{2}}$ & 0 & -0.22 & 0.22 & 0.23 & 0.76 \\
& 0 & -0.17 & 0.17 & 0 & 0.29 \\
\hline
\end{tabular}

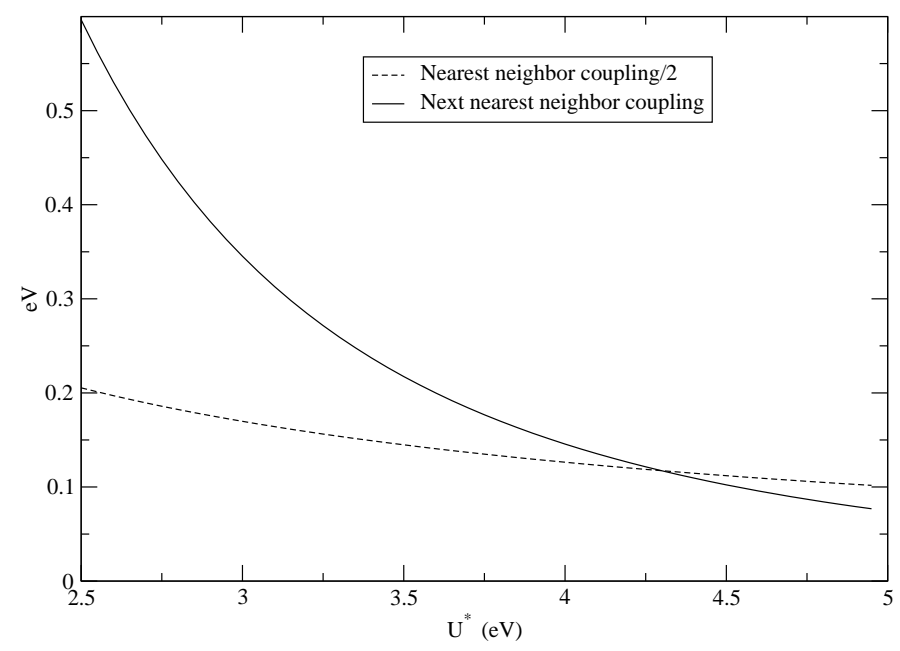

FIG. 6: The calculated nn $J_{x z, x z} / 2$ is compared to the nnn $J_{x z, x z}^{\prime}$ as a function of $U^{*}$ (using $U^{*}=U$ ). The condition for the instability of the $(\pi, \pi)$ order to the columnar order is $J_{x z, x z}^{\prime}>J_{x z, x z} / 2$ which occurs for values of $U^{*} \leq 4.3 \mathrm{eV}$.

coupling $J^{\prime}$ becomes greater than $J / 2$ for any value of $U^{*} \leq 4.3 \mathrm{eV}$ (using $U^{*}=U$ )(See Fig. 6). When $J<2 J^{\prime}$, the observed columnar antiferromagnetic ordering is favorable ${ }^{21,25}$ relative to the familiar $(\pi, \pi)$ antiferromagnetic order. The subspace spanned by $d_{x^{2}-y^{2}}$ has net spin zero and, therefore, is not expected to contribute significantly to the choice of magnetic order. On the other hand, there seems to be less frustration in the subspace spanned by $d_{x y} / d_{z^{2}}$, which is half-filled; we therefore expect that a long-range antiferromagnetic order should characterize the ground state of this subspace if it were uncoupled from the $d_{x z} / d_{y z}$ subspace. However, due to Hund's rule coupling $J^{H}$, the spin orientation of all subspaces should be common. The conflicting prefer- ences of these two subspaces, which are forced to make a common choice, introduces further frustration of relative spin orientation.

We expect that the subspace spanned by $d_{x z} / d_{y z}$ drives the system to a global columnar order $20,21,25$ because it is characterized by the larger couplings. The presence of a large $J^{\prime}$ in the subspace spanned by $d_{x z} / d_{y z}$ might impose the observed columnar order through the relatively large Hund's rule coupling $J_{H} \sim 0.5 \mathrm{eV}$. The fact that the subspace $d_{x z} / d_{y z}$ prefers the columnar order and the subspace $d_{z^{2}} / d_{x y}$ prefers the $(\pi, \pi)$ order creates frustration which may also explain the fact that the observed moment per Fe atom is small.

We emphasize that unlike the case of undoped cuprates, the undoped parent compound in the case of the oxypnictides is not an insulator. As can be inferred from Fig. 1 the motion along the ferromagnetic direction is not hindered and, therefore, the undoped material is expected to demonstrate anisotropic transport in the SDW phase.

We would like to discuss the case of the neutral FeAs layer which was considered in Sec. II where it was found that the ground state is characterized by $(\pi, \pi)$ order. The case of the neutral layer has five electrons per $\mathrm{Fe}$ atom and this implies that all Fe $d$ orbitals should be singly occupied. Therefore, the subspace spanned by $d_{x^{2}-y^{2}}$ is no longer characterized by spin zero. This means that the subspace $d_{x z} / d_{y z}$ in order to drive the columnar order has to compete against not just one but two subspaces which prefer the $(\pi, \pi)$ order.

The phenomenological hamiltonian considered in Ref. 20, 21 and in Ref. 25 to introduce frustration, is different from the one we derived based on a more rigorous approach, which is more complex. The next step would be to study the hamiltonian given in Eqs. (42), (43) by various analytical and numerical techniques, which is beyond the scope of the present work.

While the estimated $\mathrm{nn}$ antiferromagnetic coupling constants are of similar magnitude to the one in the cuprous oxides ${ }^{38}$, in the oxypnictide materials there is magnetic frustration mainly due to the fact that the nnn antiferromagnetic coupling for the $d_{x z} / d_{y z}$ subspace is large. Therefore, assuming that the pairing interaction between electrons is of magnetic origin, it is not clear if the pairing energy scale is larger or smaller compared to that in the cuprate superconducting materials. The pairing energy scale in the present model may be enhanced by the "flavor" factor, that is, the number of states spanning the subspace where the added electrons go in the case of electron doping, and by the fact that the hopping and spin-exchange matrix elements are estimated to be somewhat larger compared to those in the case of the cuprates (see Tables IXIXIXI|XII). Therefore, it is conceivable that this new class of superconductors could lead to higher critical temperatures upon future optimization of the doping agents and other factors.

A very important difference between the oxypnictides and the cuprates is that the five-fold sector can be 
thought of as formed by three subsectors, two 2-flavor sectors and a third 1-flavor sector. The $d_{x z} / d_{y z}$ sector prefers the SDW order depicted in Fig. 11, the sector spanned by the $d_{x^{2}-y^{2}}$ orbital has spin zero and the other sector spanned by $d_{x y} / d_{z^{2}}$ prefers antiferromagnetic long-range order. These subspaces are coupled by Hund's rule which, we believe, leads to the SDW order with ferromagnetic order along one direction and antiferromagnetic ordering between such chains. As in the case of cuprates, superconductivity in the oxypnictide materials might coexist with SDW order ${ }^{39}$ but these are expected to be to some extent competing orders as found in neutron 40 and $\mu S R$ studies 41 done on the superconducting doped materials.

\section{Acknowledgments}

We would to thank B. Halperin, E. Demler and S. Sachdev for useful discussions and $\mathrm{C}$. Xu for useful comments on the manuscript.
1 Y. Kamihara, H. Hiramatsu, M. Hirano, R. Kawamura, H. Yanagi, T. Kamiya, and H. Hosono, J. Am. Chem. Soc. 128, 10012 (2006).

${ }^{2}$ Y. Kamihara, T. Watanabe, M. Hirano and H. Hosono, J. Am. Chem. Soc. 130, 3296 (2008).

3 Z.-A. Ren, J. Yang, W. Lu, W. Yi, G.-C. Che, X.-L. Dong, L.-L. Sun, Zhong-Xian Zhao, Mat. Res. Innov. 12, 105 (2008) and Chin. Phys. Lett. 25, 2215 (2008).

4 X. H. Chen, T. Wu, G. Wu, R. H. Liu, H. Chen, D. F. Fang, Nature 453,761 (2008).

${ }^{5}$ G. F. Chen, Z. Li, D. Wu, G. Li, W. Z. Hu, J. Dong, P. Zheng, J. L. Luo, N. L. Wang, Phys. Rev. Lett. 100, 247002 (2008).

6 Hai-Hu Wen, Gang Mu, Lei Fang, Huan Yang, Xiyu Zhu, Europhys. Lett. 82, 17009 (2008).

7 D. J. Singh and M.-H. Du, Phys. Rev. Lett. 100, 237003 (2008).

8 I.I. Mazin, D. J. Singh, M. D. Johannes, and M. H. Du, Phys. Rev. Lett. 101, 057003 (2008).

9 K. Haule, J. H. Shim, and G. Kotliar, Phys. Rev. Lett. 100, 226402 (2008).

10 L. Boeri, O. V. Dolgov, A. A. Golubov, Phys. Rev. Lett. 101, 026403 (2008).

11 C. Cao, P. J. Hirschfeld and H.-P Cheng, Phys. Rev. B 77, 220506 (2008).

12 A.O. Shorikov, M.A. Korotin, S.V. Streltsov, S.L. Skornyakov, D.M. Korotin, V.I. Anisimov, arXiv:0804.3283.

13 Q. Si and E. Abrahams, arXiv:0804.2480

14 E. Z. Kurmaev, R. G. Wilks, A. Moewes, N. A. Skorikov, Yu. A. Izyumov, L. D. Finkelstein, R. H. Li, X. H. Chen, arXiv:0805.0668

15 T. Miyake and F. Aryasetiawan, Phys. Rev. B 77, 085122 (2008).

16 B.-L. Yu, J.C.F. Wang, A.D. Beyer, M.L. Teague, J.S.A. Horng, S.-P. Lee, N.-C. Yeh, arXiv:0804.4028.

17 K. Kuroki S. Onari, R. Arita, H. Usui, T=Y. Tanaka, H. Kontani and H. Aoki, Phys. Rev. Lett. 101, 087004(2008)

18 Zi-Jian Yao, Jian-Xin Li, Z. D. Wang, arXiv:0804.4166.

19 Xiao-Liang Qi, S. Raghu, Chao-Xing Liu, D. J. Scalapino, Shou-Cheng Zhang, arXiv:0804.4332.

20 T. Yildirim, Phys. Rev. Lett. 101, 057010(2008).

21 Fengjie Ma, Zhong-Yi Lu, Tao Xiang, arXiv:0804.3370v2

22 S. Raghu, X.-L. Qi, C.-X. Liu, D. J. Scalapino and S.-C.
Zhang, Phys. Rev. B 77, 220503(2008).

${ }^{23}$ P. A. Lee and X.-G. Wen, arXiv:0804.1739.

${ }^{24}$ V. Cvetkovic and Z. Tesanovic, arXiv:0804.4678

25 C. Xu, M. Mueller, and S. Sachdev, Phys. Rev. B 77, 020501(R)(2008).

26 Z.-Y. Weng, arXiv:0804.3228.

27 J. Li and Y. Wang, Chin. Phys. Lett. 25, 2232 (2008).

${ }^{28}$ K. Haule and G. Kotliar, arXiv:0805.0722,

29 J. Dong, H. J. Zhang, G. Xu, Z. Li, G. Li, W. Z. Hu, D. Wu, G. F. Chen, X. Dai, J. L. Luo, Z. Fang, N. L. Wang, Europhys. Lett., 83, 27006 (2008).

30 Clarina de la Cruz, Q. Huang, J. W. Lynn, Jiying Li, W. Ratcliff II, J. L. Zarestky, H. A. Mook, G. F. Chen, J. L. Luo, N. L. Wang, Pengcheng Dai, Nature 453, 899 (2008).

31 H.-H. Klauss, H. Luetkens, R. Klingeler, C. Hess, F.J. Litterst, M. Kraken, M. M. Korshunov, I. Eremin, S.-L. Drechsler, R. Khasanov, A. Amato, J. Hamann-Borreo, N. Leps, A. Kondrat, G. Behr, J. Werner, B. Buechner, Phys. Rev. Lett. 101077005 (2008).

32 J. M. Soler, E. Artacho, J. D. Gale, A. García, J. Junquera, P. Ordejón, and D. Sánchez-Portal, J. Phys.: Condens. Matter. 14, 2745 (2002).

33 N. Troullier, and J. L. Martins, Phys. Rev. B 43, 1993 (1991).

34 D. M. Ceperley, B. J. Alder, Phys. Rev. Lett. 45, 566 (1980).

35 G. Kress, J. Hafner, Phys. Rev. B 54, 11169 (1996).

36 P. E. Blöchl, Phys. Rev. B 50, 17953 (1994).

37 J. H. Jefferson, J. Phys. C 21, L193 (1988).

38 E. Manousakis, Rev. Mod. Phys. 63, 1 (1991).

39 A. J. Drew, F. L. Pratt, T. Lancaster, S. J. Blundell, P. J. Baker, R. H. Liu, G. Wu, X. H. Chen, I. Watanabe, V. K. Malik, A. Dubroka, K. W. Kim, M. Roessle, C. Bernhard, arXiv:0805.1042

40 Y. Qiu, M. Kofu, Wei Bao, S.-H. Lee, Q. Huang, T. Yildirim, J. R. D. Copley, J. W. Lynn, T. Wu, G. Wu, X. H. Chen, Phys. Rev. B 78, 052508 (2008).

41 J.P. Carlo, Y.J. Uemura, T. Goko, G.J. MacDougall, J.A. Rodriguez, W. Yu, G.M. Luke, Pengcheng Dai, N. Shannon, S. Miyasaka, S. Suzuki, S. Tajima, G.F. Chen, W.Z. Hu, J.L. Luo, N.L. Wang, arXiv:0805.2186v1. 